\title{
Comparative study of HPV16 integration in cervical lesions between ethnicities with high and low rates of infection with high-risk HPV and the correlation between integration rate and cervical neoplasia
}

\author{
LILI HAN ${ }^{1,2}$, TUERXUNAYI MAIMAITIMING ${ }^{2}$, SULAIYA HUSAIYIN ${ }^{2}$, LIN WANG $^{2}$, \\ KUNDUOZI WUSAINAHONG ${ }^{2}$, CHUNHUA MA ${ }^{2}$ and MAYINUER NIYAZI ${ }^{2}$ \\ ${ }^{1}$ Postgraduate College of Xinjiang Medical University, Urumqi, Xinjiang 830011; ${ }^{2}$ Department of Gynecology, \\ People's Hospital of Xinjiang Uygur Autonomous Region, Urumqi, Xinjiang 830054, P.R. China
}

Received October 16, 2014; Accepted August 10, 2015

DOI: $10.3892 / \mathrm{etm} .2015 .2740$

\begin{abstract}
The etiology of a high incidence of cervical cancer in populations with a low human papillomavirus (HPV) infection rate is unclear. The current study aimed to investigate the role of HPV16 DNA integration in cervical lesions in women of Han and Uygur ethnicity and to explore the association between viral integration and a high cervical cancer morbidity with a low HPV infection rate. DNA was extracted from the biopsy specimens of cervical lesions of 379 patients of Uygur ethnicity and 464 patients of Han ethnicity, and multiple quantitative polymerase chain reaction (qPCR) assays were performed to determine the copy numbers of the HPV16 E2 and E6 genes. The copy number of the HPV16 DNA was evaluated according to the E2/E6 ratio. Among these cases, 122 Uygur and 121 Han specimens were found to be HPV16 positive. In the two populations, the percentage of cases with HPV16 integration (the sum of integrated-type infection only or a mixture of free-and integrated-type infection) increased with the grade of the cervical lesions $(\mathrm{P}<0.001)$. Within groups with the same cervical lesion grade, no significant differences in HPV16 integration were found between women of Uygur and Han ethnicity (rank sum test, $\mathrm{P}>0.05$ ). No significant differences in the distribution of the HPV16 integration rate according to lesion grade were found in either population $(\mathrm{P}>0.05)$. When the two subpopulations were considered as one sample population, the integration rate significantly increased with lesion grade $(\mathrm{P}=0.02)$. These results indicate
\end{abstract}

Correspondence to: Professor Mayinuer Niyazi, Department of Gynecology, People's Hospital of Xinjiang Uygur Autonomous Region, 91 Tianchi Road, Urumqi, Xinjiang 830054, P.R. China E-mail: hanlili29@163.com

Key words: Uygur, cervical lesion, HPV16, E2 integration, multiple quantitative polymerase chain reaction that the integration rate of HPV16 E2 may serve as a molecular biological marker for the development of cervical lesions.

\section{Introduction}

Cervical cancer is the second most common malignant tumor in women throughout the world, ranking just behind breast cancer (1). The essential pathogenic factor in cervical cancer is the persistence of high-risk human papillomavirus (HPV), which is a double-stranded DNA virus with a capsid (2).

To date, numerous studies have reported on the integration of HPV DNA into the host genome in precancerous uterine cervical lesions and cervical cancers (3). High-risk HPV DNA integration into the host genome is a key factor eliciting malignant transformation in cervical lesions; both the HPV16 integration rate and the persistence of HPV16 infection demonstrate a positive correlation with the cervical lesion grade (4). Although the integration rates of HPV16 and HPV18 DNA increase with an increase in the malignancy level, the mixed type, that is, cases in which both free-type and integrated-type infection is present, is much more frequently observed in HPV16 integration. The integration of HPV16 and HPV18 into the host genome is an early step in cervical tumor development, and the incidence rate of HPV DNA integration increases with the aggravation of cervical lesions (5). HPV DNA integration has been detected in $7.4 \%$ of lesion tissues in HPV16-positive precancerous lesions of the uterine cervix, but integration occurs only in severe cervical lesions (6). These findings indicate that integration is an essential event in the progression of cervical lesions.

The Xinjiang Uygur autonomous region of China is an area with high morbidity from cervical cancer but a low HPV infection rate. The infection rate with high-risk HPV has been reported to be as low as $7.25 \%$ in women of Uygur ethnicity in Xinjiang Hotan Prefecture (7-9), compared with $12.1 \%$ in women of Han ethnicity (10). By contrast, the cervical cancer morbidity rate in women of Uygur ethnicity has been reported to be as high as 526 per 100,000 (11). The cause of the high morbidity but low HPV infection rate in Uygur women is 
unclear, and the pathogenesis of HPV remains under investigation. Loss of the E2 gene and viral gene integration into the host genome elicits the progression of precancerous lesions from low grade to high grade, which increases the risk of cervical cancer $(3,12,13)$. It may be questioned whether viral integration occurs at earlier stages in lesions in HPV16-infected women of Uygur ethnicity, whether the probability of integration is higher in Uygur women than in Han women with the same grade of lesion, and whether DNA fragments are more likely to break in women of Uygur ethnicity than in those of Han ethnicity. These questions motivated this comparative study.

Since the majority of previous studies have focused on the association of HPV16 integration with the development of cervical cancer (14-19), in the present study, HPV16-positive women were included as the subjects. A multiple quantitative polymerase chain reaction (qPCR) assay was performed to determine the copy numbers of HPV16 E2 and E6 in cervical cancer tissues and precancerous lesions. The physical state of the virus indicated by the E2/E6 ratio was then used to compare the HPV16 DNA integration rate in different grades of cervical lesions and to explore the correlation between integration and cervical lesion grade. Additionally, the HPV16 integration rates in patients of Uygur and Han ethnicity were compared to explore the role of HPV16 integration in different cervical lesion grades in women of different ethnicities.

\section{Materials and methods}

Patients and specimens. The specimens were biopsy or surgical specimens from 379 women of Uygur ethnicity and 464 women of Han ethnicity with cervical lesions treated in the Department of Gynecology of the People's Hospital of Xinjiang Uygur Autonomous Region from August 2010 to March 2011. The specimens were reviewed by two pathologists with high academic positions. No radiotherapy or chemotherapy was administered prior to biopsy or surgery. Pregnancy, immunologic insufficiency and cervical surgical history were the exclusion criteria. The specimens were stored at $-80^{\circ} \mathrm{C}$ prior to analysis. The ages of the patients of Uygur ethnicity ranged from 24 to 67 years, with a mean of $50 \pm 12$-years. The patients of Han ethnicity ranged in age from 24 to 81-years, and had a mean age of $46 \pm 14$-years. The lesion types of the patients' specimens are presented in Table I.

Approval for this study was obtained from the Institutional Review Board of the People's Hospital of Xinjiang Uygur Autonomous Region (Xinjiang, China). All participants provided written informed consent.

According to $\mathrm{Wu}$ et al (20), cervical intraepithelial neoplasia (CIN) is pathologically characterized by cervical dysplasia and carcinoma in situ with nuclear heterogeneity such as large, deeply stained nucleus with different sizes and appearance and maldistributed chromatins. Based on the degrees of cell changes and the scales of heterotypical cells, the following grades may be classified: 1, Mild atypical hyperplasia (CINI), in which cells show a mild degree of heterogeneity in an untidy arrangement but with polarity and the abnormally proliferated cells are confined to the lower $1 / 3$ part of the epithelial layer; 2, moderate atypical hyperplasia (CINII), in which cells show noticeable heteromorphism in a disordered arrangement and the abnormally proliferated cells occupy the lower 2/3 part of the epithelial layer; and 3, severe atypical hyperplasia and carcinoma in situ (CINIII), in which cells show noticeable heteromorphism without polarity and the abnormally proliferated cells occupy the lower $2 / 3$ part of the epithelial layer or even expand to the whole layer. Squamous cell carcinoma (SCC) refers to the condition in which the moistening interstitium of the focus exceeds the carcinoma in situ, showing mesh- or mass-like confluent invasion, and the pathological characteristics of cervicitis $(\mathrm{N})$ may include cervical polyp, cervical hypertrophy and Nabothian cysts.

Reagents. The TIANamp Genomic DNA kit for genomic DNA extraction from blood, cells and tissue (spin column type) was purchased from Tiangen Biotech (Beijing, China). Primers and probes for HPV16 E2 and HPV16 E6 were synthesized by Takara Biotechnology (Dalian, China) and are listed in Table II. The real-time PCR SuperMix kit, containing Premix Ex Taq ${ }^{\mathrm{TM}}$ and ROX reference dye, was purchased from Takara Biotechnology. The pCR ${ }^{\circledR}-\mathrm{XL}-\mathrm{TOPO}^{\circledR} 3.5$ kb HPV16 plasmid and SiHa cell line DNA were gifts from Xinjiang Key Laboratory of Biological Resources and Genetic Engineering, Xinjiang University (Xinjiang, China).

Extraction of the sample DNA. DNA was extracted from the specimens according to the instructions provided by the manufacturer of the TIANamp Genomic DNA kit for genomic DNA extraction from blood, cells and tissue. Clinicopathological detection was performed with a nucleic acid spectrophotometer (Beckman DU 700; Beckman Coulter, Inc., Brea, CA, USA).

Preparation of standard samples. The copy number was determined using the following formula: Copy number = weight $(\mathrm{g})$ x $6.23 \times 10^{23} / 324.5 \times 2 \times$ length of plasmid DNA (where $6.23 \times 10^{23}$ represents the molecular number of $1 \mathrm{~mol}$ of substance and 324.5 is the average molecular weight of a basic group). The copy number of the $\mathrm{pCR}^{\circledR}-\mathrm{XL}-\mathrm{TOPO}{ }^{\circledR} 3.5 \mathrm{~kb}$ HPV16 plasmid was determined to be $4.8 \times 10^{10}$ copies $/ \mu 1$, and the plasmid was then stored at $-20^{\circ} \mathrm{C}$. When adding the samples to the PCR mixes, the concentration of the plasmid was diluted to $4.8 \times 10^{10}$, $4.8 \times 10^{9}, 4.8 \times 10^{8}, 4.8 \times 10^{7}, 4.8 \times 10^{6}, 4.8 \times 10^{5}, 4.8 \times 10^{4}$ and $4.8 \times 10^{3}$ copies $/ \mu 1$ to serve as standard samples.

Determination of the E2 and E6 copy number through multiple $q P C R$. The mixes for qPCR were prepared with the following

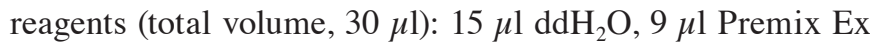
$\mathrm{Taq}^{\mathrm{TM}}$ (2X), $0.6 \mu 1 \mathrm{ROX}$ reference dye (50X), $0.6 \mu 1$ HPV16 E2 forward primer $(10 \mu \mathrm{M}), 0.6 \mu \mathrm{l}$ HPV16 E2 reverse primer (10 $\mu \mathrm{M}), 0.6 \mu 1$ HPV16 E6 forward primer $(10 \mu \mathrm{M}), 0.6 \mu 1$ HPV16 E6 reverse primer $(10 \mu \mathrm{M}), 0.5 \mu 1$ HPV16E2 Taq Man probe $(20 \mu \mathrm{M}), 0.5 \mu \mathrm{l}$ HPV16E6 Taq Man probe $(20 \mu \mathrm{M})$, and $2 \mu 1$ template DNA. The concentration of each standard DNA sample was measured three times, and the concentrations of the specimen DNA samples were measured twice. DNA extracted from $\mathrm{SiHa}$ cells was used as the negative sample control, the prepared standard sample of $4.8 \times 10^{7}$ copies $/ \mu 1$ was used as the positive system control, and a DNA blank was used as the negative system control. The PCR amplification was performed in an ABI 7500 Real-Time PCR system (Applied Biosystems Life Technologies, Foster City, CA, USA) with the following reaction conditions: Initial denaturation at $95^{\circ} \mathrm{C}$ for $30 \mathrm{sec}$ followed 
Table I. Lesion types of the specimens from women of Uygur and Han ethnicity.

\begin{tabular}{lcccccr}
\hline Ethnicity & Inflammation & CIN I & CIN II & CIN III & Cervical cancer & Total \\
\hline Uygur & 190 & 96 & 12 & 30 & 51 & 379 \\
Han & 250 & 113 & 14 & 50 & 37 & 464 \\
\hline
\end{tabular}

CIN, cervical intraepithelial neoplasia.

Table II. Primers and probes for quantitative polymerase chain reaction.

\begin{tabular}{lllc}
\hline Amplified fragments & Primers/probes & \multicolumn{1}{c}{ Sequence $\left(5^{\prime} \rightarrow 3^{\prime}\right)$} & Size (bp) \\
\hline HPV16 E2 (nt 3452-3590) & Forward primer & GAAACACAGACGACTATCCA & 195 \\
& Reverse primer & TCCGTCCTTTGTGTGAGCTGT & 132 \\
& Probe & HEX-CCAAGACAGAGCCAGACAC-Eclipse & \\
HPV16 E6 (nt 188-382) & Forward primer & GAATGTGTGTACTGCAAGCA & \\
& Reverse primer & GTTGTATTGCTGTTCTAATGTTGT & \\
& Probe & FAM-CAGCATATGGATTCCCATCTC-Eclipse & \\
\hline
\end{tabular}

HPV, human papillomavirus; nt, nucleotide.

Table III. Case population and rate of HPV16-positive cervical lesions according to lesion type in women of Uygur and Han ethnicity [n, (\%)].

\begin{tabular}{lcccccr}
\hline Ethnicity & Inflammation & CIN I & CIN II & CIN III & Cervical cancer & Total \\
\hline Uygur & $20(10.5)$ & $32(33.3)$ & $6(50.0)$ & $20(66.7)$ & $44(86.3)$ & 122 \\
Han & $22(8.8)$ & $34(30.1)$ & $7(50.0)$ & $28(56.0)$ & $30(81.1)$ & 121 \\
\hline
\end{tabular}

HPV, human papillomavirus; CIN, cervical intraepithelial neoplasia. $\chi^{2}=4.211, \mathrm{P}=0.3782$.

by $\mathrm{PCR}$ at $95^{\circ} \mathrm{C}$ for $5 \mathrm{sec}, 58^{\circ} \mathrm{C}$ for $34 \mathrm{sec}$, and $72^{\circ} \mathrm{C}$ for $30 \mathrm{sec}$ (for 40 cycles).

Statistical analysis. Samples with HPV16 E6 copy numbers $>0$ were considered HPV16 positive. The numbers of cases with cervical lesions in the different populations were expressed as an absolute number and percentages, and the HPV16 integration rates were expressed as the mean \pm standard deviation. The data were processed using SPSS software, version 17.0 (SPSS, Inc., Chicago, IL, USA). The HPV16-positive rates of the different populations were compared using the $\chi^{2}$ test, the HPV16 integration states in the various cervical lesion grades of the different populations were compared with the Kruskal-Wallis $\mathrm{H}$ test, and the integration states and integration ratios within the same cervical lesion grade in the different populations were compared using the Wilcoxon test. Differences of $\mathrm{P}<0.05$ were considered statistically significant, with a test power of 0.8 .

\section{Results}

Clinicopathological information regarding HPV16-positive patients. Detection with a nucleic acid spectrophotometer indicated that the extracted DNA had a concentration $>150 \mathrm{ng} / \mu \mathrm{l}$.
The results from the double PCR detection of HPV16 DNA in the patients are listed in Table III. The Uygur group and the Han group did not show any differences in the grades of the cervical lesions.

Standard curves. Standard curves were established based on the multiple qPCR data for E2 and E6 in the standard samples (Fig. 1). The equations used to establish the standard curves for $\mathrm{E} 2$ and $\mathrm{E} 6$ were: $\mathrm{Ct}=-3.34 \log \mathrm{X}_{0}+40.461$ and $\mathrm{Ct}=-3.417 \log \mathrm{X}_{0}+40.521$, where $\mathrm{X}_{0}$ is the original copy number and $\mathrm{Ct}$ represents the cycle threshold reflecting the number of cycles required for the fluorescence intensity in the tube to reach the designated threshold. Based on the $\mathrm{Ct}$ values for each tube, the E2 and E6 copy numbers were calculated, and $20 \mathrm{E} 2 / \mathrm{E} 6$ ratios were obtained. Of note, the E6 gene was not detected in one low-concentration standard sample. The $95 \%$ confidence interval for the $20 \mathrm{E} 2 / \mathrm{E} 6$ ratios was calculated to be $0.81-1.20$. The critical value of 0.81 was confirmed as the demarcation between HPV16 free-type infections and integrated-type infections. Specifically, E2/E6=0 indicated an integrated-type infection, E2/E6 $>0.81$ indicated a free-type infection, and $0<\mathrm{E} 2 / \mathrm{E} 6<0.81$ indicated a mixed-type infection (both free-type and integrated-type). 
Table IV. Comparison of the integration states of HPV16 [n, (\%)].

\begin{tabular}{|c|c|c|c|c|c|c|c|c|}
\hline \multirow[b]{2}{*}{ Lesion grade } & \multicolumn{3}{|c|}{ Uygur $(n=122)$} & \multicolumn{3}{|c|}{$\operatorname{Han}(n=121)$} & \multirow[b]{2}{*}{$\mathrm{Z}^{\mathrm{a}}$} & \multirow[b]{2}{*}{ P-value } \\
\hline & Free & Mixed & Integrated & Free & Mixed & Integrated & & \\
\hline $\mathrm{N}$ & $16(13.11)$ & $4(3.28)$ & $0(0)$ & $15(12.40)$ & $7(5.79)$ & $0(0)$ & -0.86 & 0.39 \\
\hline CIN I & $19(15.57)$ & $13(10.66)$ & $0(0)$ & $17(14.05)$ & $17(14.05)$ & $0(0)$ & -0.76 & 0.45 \\
\hline CIN II/III & $8(6.56)$ & 17 (13.93) & $1(0.82)$ & $9(7.44)$ & $25(20.66)$ & $1(0.83)$ & -0.34 & 0.73 \\
\hline $\mathrm{SCC}$ & $9(7.36)$ & $32(26.23)$ & $3(2.46)$ & $7(5.79)$ & $21(17.36)$ & $2(1.65)$ & -0.26 & 0.80 \\
\hline$\chi^{2 b}$ & & 26.18 & & & 16.14 & & & \\
\hline P-value & & 0.00 & & & 0.00 & & & \\
\hline
\end{tabular}

${ }^{a}$ Wilcoxon test; ${ }^{b}$ Kruskal-Wallis H test. HPV, human papillomavirus; N, negative; CIN, cervical intraepithelial neoplasia; SCC, squamous cell carcinoma.

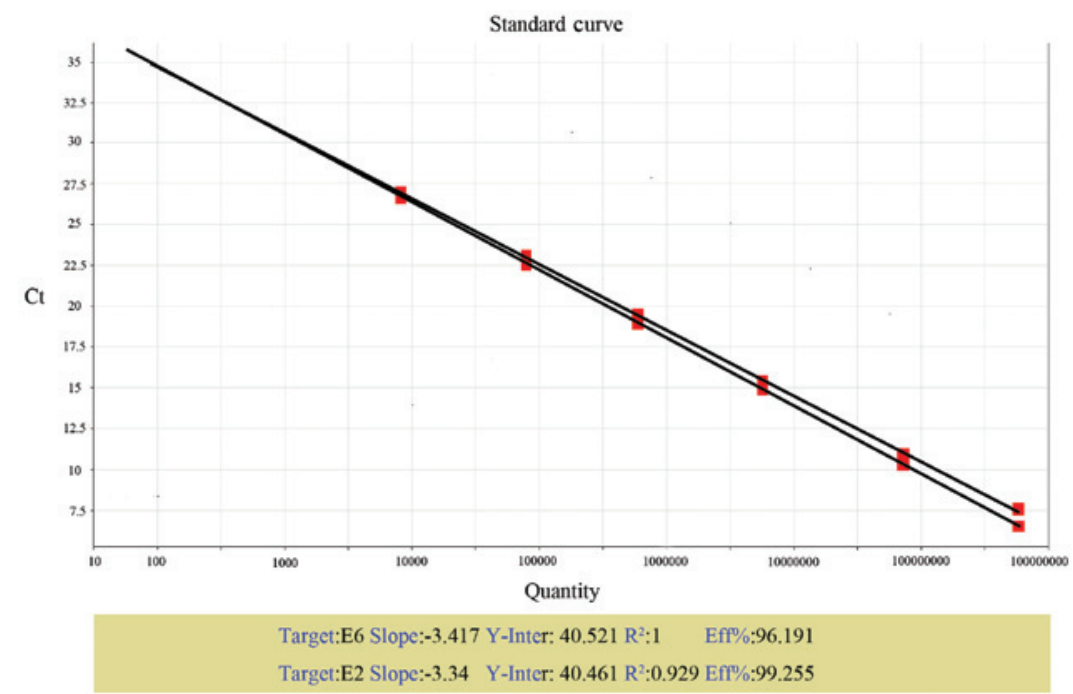

Figure 1. Standard curve for the standard samples based on multiple quantitative polymerase chain reaction results.

HPV16 integration rates in different grades of cervical lesions. The HPV16 integration ratios in cervical lesions of different grades were detected. In the positive system control, the ratio of E2/E6 was 0.96. E2 and E6 were not detected in the negative system control. In the negative sample control, the ratio of E2/E6 was 0. The integration state of HPV16 in the patients of Uygur and Han ethnicity is shown according to lesion grade (N, CIN I, CIN II/III or SCC) in Table IV. According to the Kruskal-Wallis $\mathrm{H}$ test for multi-sample comparisons, the type distribution within the same population showed a significant difference $(\mathrm{P}<0.05)$; specifically, as the lesion grade increased, the proportion of free-type HPV16 infections decreased, and the proportion of integrated-type infections increased. Between the Uygur and Han populations, the proportions of integration showed no significant differences within the same cervical lesion grade $(\mathrm{P}>0.05)$.

HPV16 integration rates within different cervical lesion grades in patients of Uygur and Han ethnicities. The integration rate was defined as 1-E2/E6. The HPV16 integration rates for the lesions of grades N, CIN I, CIN II, CIN III and SCC are shown in Figs. 2 and 3. In the Uygur patients with lesion grades $\mathrm{N}$ and CIN I and in the Han patients with lesion grades N, CIN I and CIN II, the integration rates were $<0.5$. In the lesions of grades N, CIN I, CIN II, CIN III and SCC in patients of either Uygur or Han ethnicity, the integration rate of HPV16 appeared to increase as the grade of the cervical lesions increased; however, the increase was not found to be statistically significant according to the Kruskal-Wallis $H$ test (Uygur: $\chi^{2}=2.44$, $\mathrm{P}=0.30$; Han: $\chi^{2}=4.12, \mathrm{P}=0.13$ ).

Between the Uygur and Han patients, there was no significant difference in the HPV16 integration rate within the same grade of lesion (Wilcoxon test of 2 independent samples, $\mathrm{P}>0.05$; Table V). Irrespective of the differences between the two populations, the Uygur and Han patients were considered as one sample to increase the sample size. As the grade of the lesions increased from N to CIN I, CIN II/III and SCC, the integration rate increased gradually, and the increase was statistically significant (Kruskal-Wallis $\mathrm{H}, \chi^{2}=8.42, \mathrm{P}=0.02$ ).

\section{Discussion}

In this study, the proportion of integration within the same cervical lesion grade demonstrated no statistically significant 


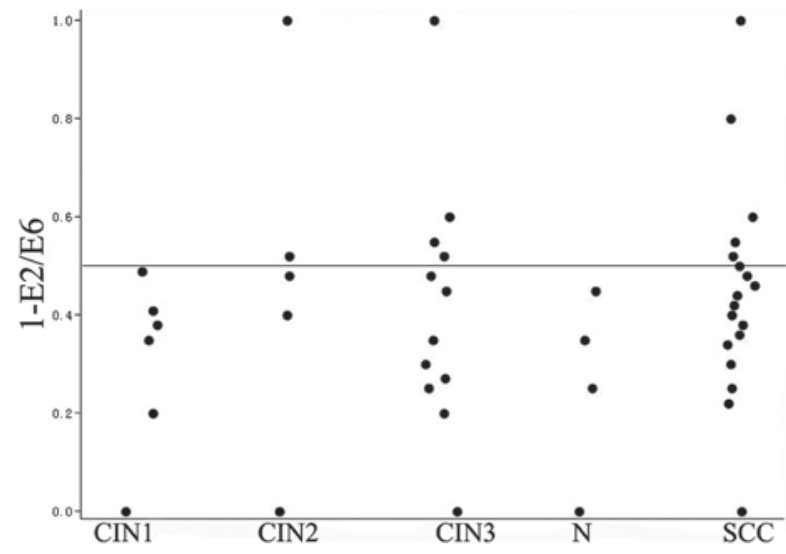

Figure 2. Distribution of the HPV16 integration rates according to cervical lesion grade in Uygur patients. HPV, human papillomavirus; CIN, cervical intraepithalial neoplasia; $\mathrm{N}$, negative; SCC, squamous cell carcinoma.

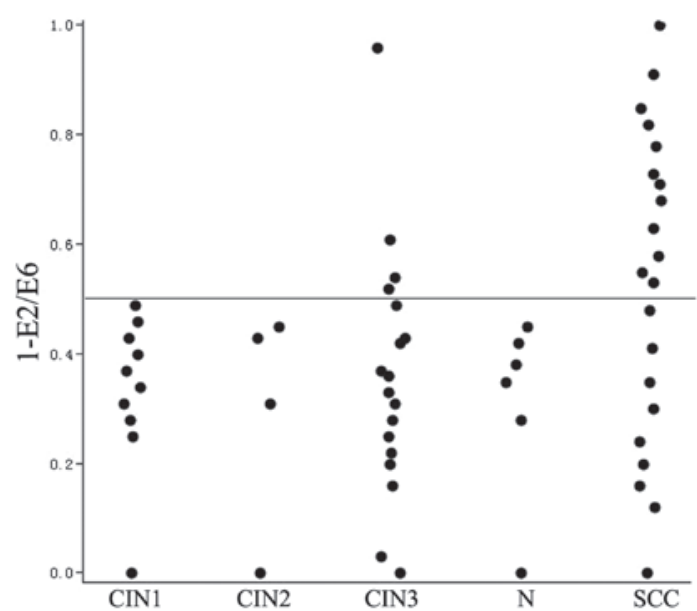

Figure 3. Distribution of the HPV16 integration rates according to cervical lesion grade in Han patients. HPV, human papillomavirus; CIN, cervical intraepithalial neoplasia; $\mathrm{N}$, negative; SCC, squamous cell carcinoma.

difference between Uygur and Han ethnicities. Even in the group of patients with CIN II/III grade lesions, the integration ratio in the women of Han ethnicity appeared to be higher than that in the women of Uygur ethnicity. Neither of these findings is able to account for the high morbidity but low HPV infection rate observed in the Xinjiang Uygur population. Based on the results of this study, there are four potential explanations. First, E2 contains an $\mathrm{N}$ terminus, $\mathrm{C}$ terminus and hinge region. In the study by Zhang et al, the miss rates for the $\mathrm{N}$ terminus, hinge region and $\mathrm{C}$ terminus were $71.88,50.00$ and $21.88 \%$, respectively (21). It may be possible that frequent fractures occur outside of the hinge region in women of Uygur and Han ethnicity in Xinjiang. This possibility is dependent on the complete detection and analysis of the fracture sites of E2, and even of E1, to identify whether actual differences in HPV16 integration rates exist between Uygur and Han women. Second, the small sample size limited the detection accuracy; thus, increasing the sample size might improve the detection power in further studies. Third, no consensus has been reached regarding the pathogenesis of HPV16 integration into the host genome during cervical cancer development. Among patients with CIN I, CIN II/III and SCC from France, the HPV16 integration rates were 64, 83.3 and
Table V. Comparison of the HPV16 integration rates between the patients of Uygur and Han ethnicity with the same cervical lesion grades (mean \pm standard deviation).

\begin{tabular}{lcccc}
\hline Grade & Uygur & Han & $\mathrm{Z}^{\mathrm{a}}$ & P-value \\
\hline $\mathrm{N}$ & $0.16 \pm 0.12$ & $0.13 \pm 0.10$ & -0.57 & 0.60 \\
CIN I & $0.27 \pm 0.18$ & $0.23 \pm 0.15$ & -0.54 & 0.59 \\
CIN II/III & $0.38 \pm 0.26$ & $0.32 \pm 0.22$ & -0.58 & 0.56 \\
SCC & $0.41 \pm 0.24$ & $0.51 \pm 0.30$ & -0.56 & 0.58 \\
\hline
\end{tabular}

${ }^{a}$ Wilcoxon test. HPV, human papillomavirus; CIN, cervical intraepithalial neoplasia; N, negative; SCC, squamous cell carcinoma.

$82 \%$, respectively. Although the integration rate increased as the grade of the lesion increased, this change was not statistically significant, which might be attributable to the small sample size (22). Another previous study of CIN and cervical cancer cases revealed that the average HPV16 integration rate was $68 \%$, and the researchers concluded that HPV16 integration is not an essential pathogenic factor in the development of cervical cancer (23). In addition, a previous study demonstrated different proportions of HPV16 integration within the same cervical lesion grade from different areas in the world (20). Finally, in women with cervical cancer, multiple infections occur more often in Uygur women than in Han women. As a carcinogenic mechanism, multiple infections might facilitate amplification of the telomerase RNA component (TERC) gene, resulting in the higher morbidity of cervical cancer in Uygur women than in Han women (24). Certainly, certain customs and conditions of the Uygur population, including early marriage, early pregnancy, multiple births, poor health habits, inconvenient transportation, insufficient medical facilities and lack of a cervical cancer screening system, may also substantially contribute to the high morbidity of cervical cancer.

The proportion of HPV16 integration can provide a cross-sectional perspective of the integration incidence in a population and might reflect the difficulty of integration in different cervical lesion grades. The integration rate can also provide a longitudinal perspective of integration and may indicate the intensity of viral integration in individuals. This latter aspect might be beneficial for disease assessments of individual patients (25) and could trigger shunting effects that affect the prognosis of HPV-positive CIN II cases (26). The results of the present study demonstrate different degrees of HPV16 integration between different grades of CIN and cervical cancer in the study population. In the N, CIN I, CIN II/III and SCC groups, the proportion of free HPV16 decreased as the lesion grade increased, but the proportion of integration (mixed- and fractured-type) increased gradually. The integrated type was found in CIN II/III and SCC lesions, and the percentage of the integrated type was highest in SCC. Specifically, as the grade of the cervical lesion increased, a larger rate of HPV16 integration was observed, which revealed that HPV16 integration into the host genome is a key pathogenic factor in cervical cancer. This finding is consistent with studies of women of Han ethnicity in Sichuan and Hubei conducted by Zheng et al (27), Shi et al (28) and Li et al (29). 
The mean integration rates in the N, CIN I, CIN II/III and SCC groups demonstrated no statistically significant differences between the Uygur and Han patients. When the differences between the populations were ignored and the Uygur and Han women were considered as one sample, it was found that the integration rate increased as the lesion grade increased from $\mathrm{N}$ to CIN I, CIN II/III and SCC to a statistically significant degree. This finding indicates that an increased sample size might help to elucidate the association between viral integration and the progression of cervical lesions. Once the association between increasing viral integration and cervical lesion progression is established, the comparison between Uygur and Han ethnicities will become significant.

A previous study detected HPV16 in cases graded as N, CIN I, CIN II/III and SCC with median HPV16 E2/E6 ratios of $0.75,0.58,0.51$ and 0.36 , respectively. The ratio tended to decrease with increasing cervical lesion grade (22). That investigation revealed that viral loads $>22,000$ copies $/ 1,000$ cells and yielding an E2/E6 ratio $<0.5$ tend to progress into higher grade lesions. This finding may guide further studies concerning the integration rate in Han women using larger sample populations.

In conclusion, HPV16 integration was found to be an essential factor contributing to the progression of cervical lesions in women of Uygur and Han ethnicity, and no differences in HPV integration were observed between the two populations. In addition, no correlation was observed between the integration rate and the progression of the cervical lesion grade. In further studies, larger sample sizes and more sensitive detection methods are required to explore the objective pathogenesis of the high cervical cancer morbidity in women of Uygur ethnicity.

\section{Acknowledgements}

The authors thank the staff of the Department of Gynecology of Xinjiang Uygur Autonomous Region People's Hospital for assistance during the study. This study was supported by the National Natural Science Foundation of China (grant no. 81160317).

\section{References}

1. Cornelison TL: Humanpapillomavirus genotype 16 vaccine for cervical cancer prophylaxis and treatment. Cirr Opin Oncol 12: 466-473, 2000

2. Muñoz N, Bosch FX, de Sanjosé S, Herrero R, Castellsagué X, Shah KV, Snijders PJ and Meijer CJ; International Agency for Research on Cancer Multicenter Cervical Cancer Study Group: Epidemiologic classification of human papillomavirus types associated with cervical cancer. N Engl J Med 348: 518-527, 2003.

3. Pett M and Coleman N: Integration of high-risk human papillomavirus: A key event in cervical carcinogenesis? J Pathol 212: 356-367, 2007.

4. Jiang SQ, Tu SA, Zhou JL, Mai M, Bi J, Ru X, Sha L, Xu X, Hai $R$ and Ai M: Investigation and analysis of gynecopathy in Cele county of Xinjiang, China. Zhongguo Fu You Bao Jian 21: 524-526, 2006 (In Chinese).

5. Li N and Dai M: Human papillomavirus in China: A multiple-centric cross sectional study. Zhonghua Ji Bing Kong Zhi Za Zhi 12: 411-415, 2008 (In Chinese).

6. Li L, Pan Q, Liu XF, Wu YP, Qiao YL, Ma Y, Chen F, Zhu K, Liu XW, RE Z, et al: A cross-sectional study: The prevalence and distribution characteristic of HPV infection in Uygur women in Xinjiang. Ai Zheng Jin Zhan 2: 114-118, 2010 (In Chinese)

7. Li J, Li LK, Ma JF, Wei LH, Niyazi M, Li CQ, Xu AD, Wang JB, Liang H, Belinson J and Qiao YL: Knowledge and attitudes about human papillomavirus (HPV) and HPV vaccines among women living in metropolitan and rural regions of China. Vaccine 27: 1210-1215, 2009.
8. Cricca M, Venturoli S, Leo E, Costa S, Musiani M and Zerbini M: Disruption of HPV $16 \mathrm{E} 1$ and E2 genes in precancerous cervical lesions. J Virol Methods 158: 180-183, 2009.

9. Li W, Wang W, Si M, Han L, Gao Q, Luo A, Li Y, Lu Y, Wang S and Ma D: The physical state of HPV16 infection and its clinical significance in cancer precursor lesion and cervical carcinoma. J Cancer Res Clin Oncol 134: 1355-1361, 2008.

10. Huang LW, Chao SL and Lee BH: Integration of human papillomavirus type-16 and type-18 is a very early event in cervical carcinogenesis. J Clin Pathol 61: 627-631, 2008.

11. Matovina M, Sabol I, Grubisić G, Gasperov NM and Grce M: Identification of human papillomavirus type 16 integration sites in high-grade precancerous cervical lesions. Gynecol Oncol 113: 120-127, 2007.

12. Tinelli A, Vergara D, Leo G, Malvasi A, Casciaro S, Leo E, Montinari MR, Maffia M, Marsigliante S and Lorusso V: Human papillomavirus genital infection in modern gynecology: Genetic and genomic aspects. Eur Clinics Obst Gynaecol 3: 1-6, 2007.

13. Arias-Pulido H, Peyton CL, Joste NE, Vargas H and Wheeler CM: Human papillomavirus type 16 integration in cervical carcinoma in situ and in invasive cervical cancer. J Clin Microbiol 44: 1755-1762, 2006.

14. Bosch FX, Manos MM, Muñoz N, Sherman M, Jansen AM, Peto J, Schiffman MH, Moreno V, Kurman R and Shah KV; International Biological Study on Cervical Cancer (IBSCC) Study Group: Prevalence of human papillomavirus in cervical cancer: A worldwide perspective. J Natl Cancer Inst 87: 796-802, 1995.

15. Pirami L, Giache V and Becciolini A: Analysis of HPV 16, 18, 31 and 35 DNA in pre-invasive and invasive lesions of the uterine cervix. J Clin Pathol 50: 600-604, 1997.

16. Kalantari M, Blennow E, Hagmar B and Johansson B: Physical state of HPV16 and chromosomal mapping of the integrated form in cervical carcinomas. Diagn Mol Pathol 10: 46-54, 2001.

17. Kristiansen E, Jenkins A and Holm R: Coexistence of episomal and integrated HPV16 DNA in squamous cell carcinoma of the cervix. J Clin Pathol 47: 253-256, 1994.

18. Park JS, Hwang ES, Park SN, Ahn HK, Um SJ, Kim CJ, Kim SJ and Namkoong SE: Physical status and expression of HPV genes in cervical cancers. Gynecol Oncol 65: 121-129, 1997.

19. Dürst M, Kleinheinz A, Hotz M and Gissmann L: The physical state of human papillomavirus type 16 DNA in benign and malignant genital tumours. J Gen Virol 66: 1515-1522, 1985.

20. Wu XX, Niyazi M, Zhu KC et al: Status of HPV16 infection in cervical lesions in Uygur women and its clinical significance. Zhong Guo Zhong Liu 21: 394-397, 2012 (In Chinese).

21. Zhang S, Cai HB and Ding XH: Different deletion states of HPV16 E2 gene in C-terminal hinge region and N-terminal in cervical lesions. Xiandai Fuchanke Jinzhan 19: 728-731, 2010 (In Chinese).

22. Saunier M, Monnier-Benoit S, Mauny F, Dalstein V, Briolat J, Riethmuller D, Kantelip B, Schwarz E, Mougin C and Prétet JL: Analysis of human papillomavirus type 16 (HPV16) DNA load and physical state for identification of hpv16-infected women with high-grade lesions or cervical carcinoma. J Clin Microbiol 11: 3678-3685, 2008.

23. Sathish N, Abraham P, Sridharan G, Shaji RV, Chandy G and Peedicayil A: E2 sequence variations of HPV 16 among patients with cervical neoplasia seen in the Indian subcontinent. Gynecol Oncol 95: 363-369, 2004.

24. Liu KJ,Liu Q and Tang HF: Study on genotypes of human papilloma virus infected Uigur and Han women with cervical cancer and their relationships with TERC gene amplification. Zhongguo Fuchanke Linchuang Zazhi 6: 449-452, 2008 (In Chinese).

25. Li HH: The study on the corralation between cervical cancer and HPV DNA integration, E2 disruption, ratio of HPV E2/E6. Zhongguo Mian Yi Xue Za Zhi, 26: 670-673, 2010 (In Chinese).

26. Li Li, Mayinuer Niyazi, Wenhua Zhang, et al: A cross-section population study on different cervical cancer screening methods in uygur women of xinjiang. Zhongguo Zhongliu 19: 238-242, 2010 (In Chinese).

27. Zheng Y, Peng ZL, Lou JY and Wang H: Detection of HPV16 integration status in preinvasive and invasive cervical carcinoma. Zhongguo Kang Ai Xie Hui 33: 961-964, 2006 (In Chinese).

28. Shi YM, Ye H, Xiao CY, Chen JL, Wang YQ and Wu JF: Change and significance of HPV integrated state in cervical lesions. Shandong Yi Yao 49: 1-3, 2009.

29. Li KZ, Jin Z, Fang Y and Ai JH, et al: Study on the correlation between integrated state of HPV-16 and the occurrence of cervical cancer. Zhongguo Fu You Bao Jian 26: 4241-4244, 2011 (In Chinese). 